\section{Schlafmangel - Einfluss auf Kommunikation und Interaktion im Team?}

Neuschwanderl A et al. Impact of sleep deprivation on anaesthesia residents' non-technical skills: a pilot simulation-based prospective randomized trial. Br J Anaesth 2017; 119: 125-131

Menschliches Versagen in der Medizin kann zu Behandlungsfehlern führen und hat somit weitreichende Konsequenzen für unsere Patienten. Nach einer Auswertung von Schadensfällen des „US Institute of Medicine“ sterben in den USA zwischen 44000-98000 Patienten jährlich an den Folgen von Behandlungsfehlern.

Besonders in der Anästhesie kann Schlafentzug durch Überlastung und Schichtdienst den perioperativen Workflow gefährden und zu mangelnder Aufmerksamkeit führen. Mehrere Studien haben den Einfluss von Schlafentzug auf die manuellen Fertigkeiten untersucht und konnten zeigen, dass sowohl Aufmerksamkeit als auch die Geschwindigkeit bei Routinetätigkeiten deutlich nachlassen. Interessanterweise war die Durchführung schwieriger Prozeduren, wie z.B. die Anlage eines PDKs als auch die Reaktion in Notfallsituation hiervon nicht beeinträchtigt.

Doch für eine strukturierte und sichere Patientenversorgung im OP sind auch Kommunikationsverhalten, Sozialkompetenz, Ressourcenausschöpfung und die Leitung eines Teams wichtig. Es müssen Entscheidungen sicher getroffen, Aufgaben verteilt und die Teamarbeit gefördert werden. Unabhängig von den manuellen Fähigkeiten eines Anästhesisten sind diese Eigenschaften in Notfallsituationen besonders gefragt. In vorausgegangenen Studien gibt es Hinweise, dass Schlafentzug die erwähnten sog. nicht technischen Fähigkeiten stark beeinflussen und eine mangelnde situative Aufmerksamkeit zu gravierenden Behandlungsfehlern führen kann. Nicht medizinische Bereiche, wie z. B. das Flugverkehrswesen und Industriebereiche mit Gefahrstoffverarbeitung, versuchen, dem erhöhten Risiko durch menschliches Versagen über zahlreiche Sicherheitsalgorithmen Rechnung zu tragen. Auch in der

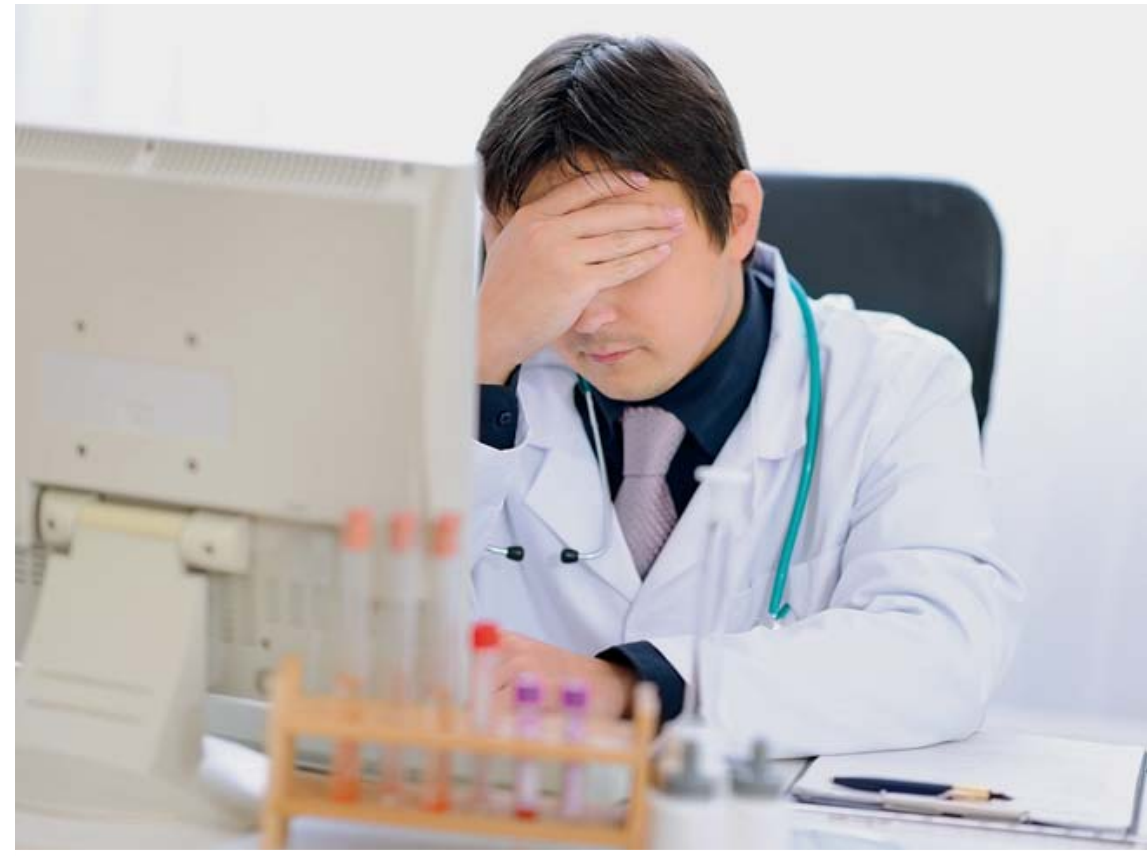

- Abb. 1 Quelle: Alliance/Fotolia.de.

Medizin werden immer mehr Notfallsituationen durch Simulation und Team-Training optimiert.

Kliniker und Wissenschaftler aus Frankreich führten mit der Hypothese, dass Schlafentzug den Abruf der erwähnten Soft Skills in simulierten Notfallsituationen verzögert, eine Pilotstudie durch. Als sekundäre Endpunkte wurden auch der Einfluss von Schlafmangel auf manuelle Fähigkeiten, Wachheit und Selbstbewusstsein untersucht.

Es wurden 20 Anästhesisten, die an akademischen Lehrkrankenhäusern in Paris tätig waren, in 2 Gruppen (Gruppe 1: Schlafentzug durch Nachtdienst, Gruppe 2: normaler Schlaf zu Hause) randomisiert. In einer Simulationsumgebung wurde eine Notfalleinleitung für eine chirurgische Laparotomie bei Peritonitis präsentiert, bei der als Komplikation ein anaphylaktischer Schock nach Gabe von Succinylcholin auftritt. Während des Notfallmanagements wurde die Situation für den Probanden zusätzlich durch das Erscheinen des chirurgischen Teams erschwert, das im Rahmen der Simulation versuchte, den Handlungsablauf durch Diskussion über das weitere
Management (Operation vs. Intensivtherapie) zu stören.

Die erbrachte Leistung in der Notfallsituation beurteilten unabhängig 2 erfahrene, für die Probandengruppe verblindete Anästhesisten über die ANTS-Skala, ein Bewertungsverfahren mit 4 Kategorien (Situationsmanagement, Teamarbeit, situative Aufmerksamkeit, Entscheidungsfindung) anhand von Videoaufzeichnungen.

Insgesamt waren die erbrachten Leistungen in der Gruppe mit ausreichend Schlaf besser als in der Gruppe mit Schlafentzug, was sich in einem im Median besseren ANTS-Wert zeigte (14,5 vs. 12,2). Interessanterweise war vor allem die Teamarbeit in der Schlafentzugsgruppe deutlich schlechter (3 vs. 4), während sich die Scores für situative Wachheit, Entscheidungsfindung und Notfallmanagement kaum voneinander unterschieden. Die Untersucher fanden zusätzlich eine erhöhte Inzidenz für Schläfrigkeit und Unsicherheit bezogen auf die durchgeführten anästhesiologischen Tätigkeiten. 


\section{FAZIT}

Trotz der Schwächen dieser Studie (kleine Gruppen, nur eine simulierte Notfallsituation) wird deutlich, dass neben einem reinen Technik-Training und Teaching die Simulation und das Trainieren der Interaktion bei Notfallsituationen sowohl für die Sicherheit unserer Patienten als auch für die Selbsteinschätzung und die Souveränität in diesen Situationen entscheidend ist. Leider wurde in der Studie ein relativ junges Probandenkollektiv (24-30 Jahre) hinsichtlich der Konsequenzen eines Schlafmangels untersucht. Erfahrungsgemäß nehmen jedoch Ruhephasen (Nachwuchs/ telefonische Erreichbarkeit/Doppelbelastung) und Kompensationsmechanismen (Sport/Schlaf/freie Tage) mit zunehmendem Alter ab. Es wäre also interessant, die Studie mit unterschiedlichen Altersklassen zu wiederholen.

Dr. med. Ralf Quabach, Solingen 